PART II

OBSERVATIONS OF SPIRAL STRUCTURE IN OUR GALAXY 
A. RADIO OBSERVATIONS 


\title{
17. SPIRAL STRUCTURE OF NEUTRAL HYDROGEN IN OUR GALAXY
}

\author{
F. J. KER R \\ University of Maryland, College Park, Md., U.S.A.
}

\begin{abstract}
This paper discusses the evidence on the hydrogen spiral structure which is available from $21-\mathrm{cm}$ observations. The problems involved in deriving a hydrogen map are discussed, and one interpretation of the spiral pattern is presented. Some of the major characteristics of the $\mathrm{HI}$ in spiral arms are discussed, and a comparison is made between the kinematics of $\mathrm{HI}$ and $\mathrm{HII}$.
\end{abstract}

\section{Introduction}

I wish to discuss some of the observational data about hydrogen in spiral arms, partly as an introductory review, and partly to present some new results. We know quite clearly that $\mathrm{H}$ i concentrates to elongated features, which are presumably spiral arms and their minor components, but the detailed pattern is far from clear. The difficulties come from:

(1) The enormous amount of fine structure detail, which makes it hard to recognize the main features, or even to know whether there is a regular pattern.

(2) As is well known, distances are kinematic, and other methods of deriving distance can only help to a small extent.

\section{Velocity-Distance Problem}

In getting kinematic distances, we assume that differential rotation is always the most important motion, and usually consider that rotation is circular and axisymmetric, or at least follows a regular pattern. A great deal of attention has been given to derivation of the best possible rotation curve. The only method available for the main part of the Galaxy is from the $21-\mathrm{cm}$ observations themselves, using the well-known method in which the extreme velocity on a line profile is considered to represent the circular velocity at the tangential point, the position on the line of sight which is closest to the galactic center. Extrapolation to the region outside the sun is commonly done through a model such as the Schmidt model, which is designed to fit the inner region results, with some assistance from optical data near the sun.

Figure 1 shows again a pair of such curves from $21-\mathrm{cm}$ observations at Parkes, giving the variation of the (assumed circular) rotational velocity in the galactic plane with distance from the center, for the first and fourth quadrants of longitude (the so-called 'northern' and 'southern' sides of the Galaxy). We see the well-known north-south asymmetry, the approximate similarity of the two curves, the large-scale oscillation which is presumably related to end-on spiral arms, and the small-scale fluctuations.

Rotation curves derived by other workers from $21-\mathrm{cm}$ observations (e.g., Shane 
and Bieger Smith (1966) in the north and Bajaja et al. (1967) in the south) agree quite well with the Parkes curves. The detailed differences that are found are probably due to the use of different beamwidths and bandwidths. There is also an interesting field of study in the differences between rotation curves at different $z$-levels with respect to the galactic plane, or the curve obtained always from the latitude where the hydrogen density is a maximum, and not restricted to the galactic plane. In the present context, however, these minor differences do not seriously affect the spiral mapping problem, where the greatest difficulty is the restriction to kinematic distances.

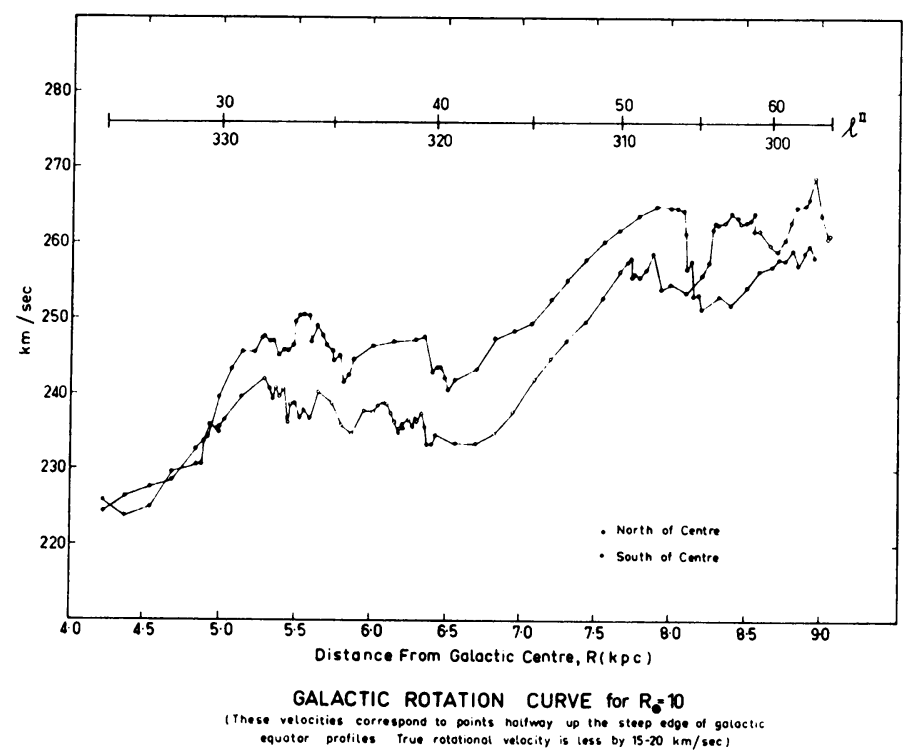

Fig. 1. Galactic rotation curves for the northern and southern sides of the center, derived from tangential point observations, assuming circular rotation (Kerr, 1964).

The north-south asymmetry shows immediately that the motion is not entirely circular; perhaps there is an oval distortion or some other large-scale effect. The velocity fluctuations are related to the fine structural details, and these interfere seriously with the possibility of drawing a precise map of the spiral structure. For any small feature, there is no way for us to distinguish between its peculiar velocity and the rotational component, and distances must be uncertain by an amount related to the peculiar or non-circular component. Such errors are proportionately greatest at short distances, where the hydrogen picture is consequently weakest, but they must be present all over the Galaxy. A further and probably related complication is that the very presence of a spiral feature distorts the velocity pattern in its vicinity, as will be discussed by some of the theoretical workers. Clearly the structural and kinematic problems must be solved together.

We cannot expect, as used to be thought, that we can ever produce a fully detailed 
map of the hydrogen structure on a kinematic basis alone. A better understanding of the large-scale velocity field should give us a reasonable picture of the major spiral arms, but accurate positions for the smallest hydrogen features will always elude us.

\section{Mapping}

Figure 2 shows one version of the hydrogen spiral pattern, projected on to the galactic plane; this is based on observations by Kerr and Hindman (Parkes) and Henderson (Green Bank). In the outer region, the Schmidt model and circular motion have been assumed. Within these limitations, a clear trailing pattern can be seen. Near the sun, a Carina-Cygnus arm is shown, but it is not clear whether this is actually continuous through the sun. As stated above, the possibility of peculiar motions makes this part of the diagram quite uncertain in detail.

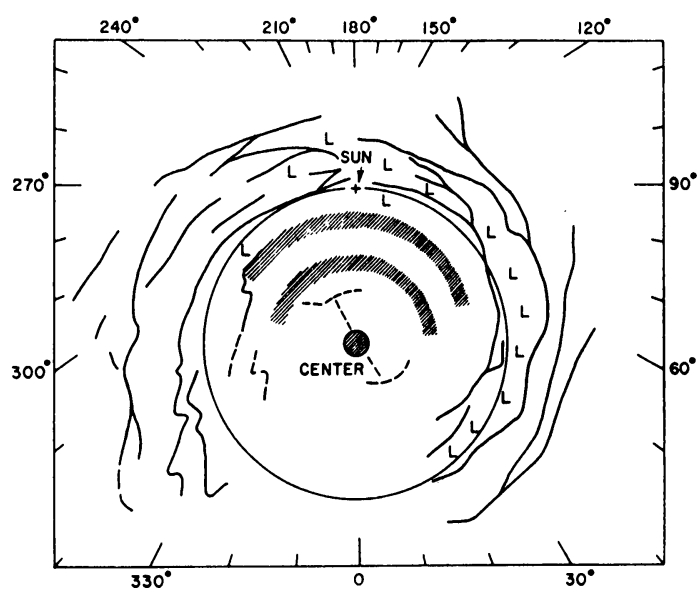

Fig. 2. Sketch of the main features of the neutral hydrogen spiral structure, from observations of Kerr (1969a), Hindman (1969) and Henderson (1967). Structural details are not shown in the inner region, owing to the large uncertainty in the distance. Regions of low hydrogen density are indicated by $\mathrm{L}$.

Inside the sun's position the two major arms, the Sagittarius arm and the NormaScutum arm, are only roughly sketched, and no attempt has been made to represent the detail in this region. Further in are the 'expanding' 3-kpc arm and a possible rudimentary bar of high-velocity gas.

The great complexity of the distribution in the inner region is clearly seen in Figure 3, due to G. Knapp, in which the identifiable features are drawn in a velocitylongitude plot, with the best estimate of their continuity. The solid-line features are at positive latitudes, and the dashed-lines are negative; the width of the lines gives an indication of the importance of each feature. The latitude characteristics of the features will be discussed in more detail later.

The Sagittarius arm, Norma-Scutum arm, and the 3-kpc arm can be clearly seen in 
the diagram, as well as another feature near the center at -70 to $-50 \mathrm{~km} \mathrm{~s}^{-1}$. Each major arm shows considerable branching and splitting, and there are suggestions of cross-linkages. It must be remembered, however, that features which are adjacent or in contact on this diagram are not necessarily near to each other in space, as their latitudes may be different and also different peculiar velocity components may give them different distances.

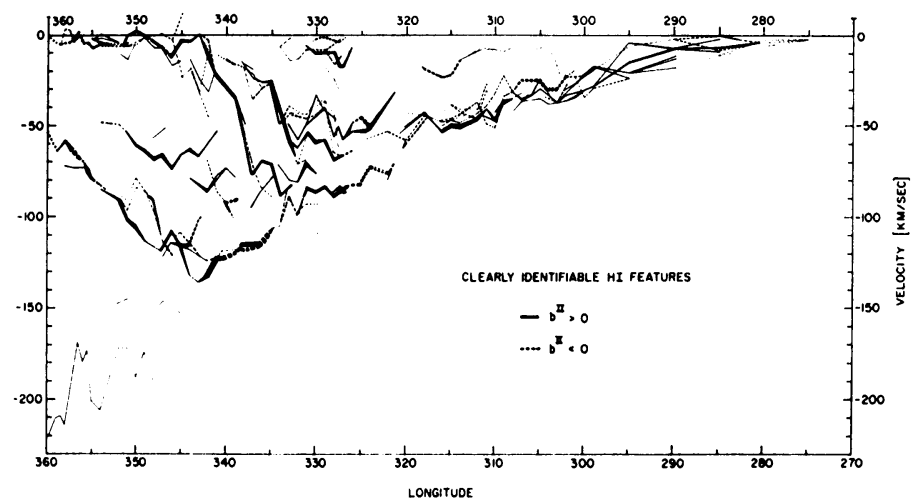

Fig. 3. Velocity-longitude plot of clearly-identifiable spiral features, with the best estimate of their continuity. The width of a line indicates the importance of the feature.

To go from this stage to a real map, i.e., to convert velocity into distance, requires a full understanding of the velocity field, and also a resolution of the velocity ambiguity for each component, to decide between the 'near' and 'far' locations. The smaller features at least must have non-circular components of velocity, and are consequently difficult to locate in position. The velocity ambiguity can sometimes be resolved, particularly for the major features, but not sufficiently completely for a good map to be drawn. There is a strong suggestion that the two major arms are curving away in a trailing sense. This can be seen in Figure 4 for the Sagittarius arm, where the wide feature at $-55 \mathrm{~km} \mathrm{~s}^{-1}$ and the narrower one at $-22 \mathrm{~km} \mathrm{~s}^{-1}$ probably belong to a single trailing arm, with a pitch angle of $8^{\circ}$. The arm trails in a similar way in the first quadrant, but the feature is not regular enough to be drawn in over its entire length.

Partial evidence on the location of the inner arms can be obtained from considerations of tangency. We saw earlier that the major oscillations in the rotation curves indicate the tangential directions of the major arms. Also, in a plot of the integrated hydrogen (Kaplan, unpublished), the Norma-Scutum arm shows up strongly in cross section on both sides, centered at about $l^{\mathrm{II}}=330^{\circ}$ and $31^{\circ}$. On the southern side, the arm appears to be centered a few tenths of a degree above the equator, while on the northern side it is centered right on the equator. The Sagittarius arm can also be seen in cross-section at $l^{\mathrm{II}}=303^{\circ}-305^{\circ}$ and at $55^{\circ}$, but less strongly than is the case for the Norma-Scutum arm.

These tangential directions also show up clearly as steps in the continuum back- 


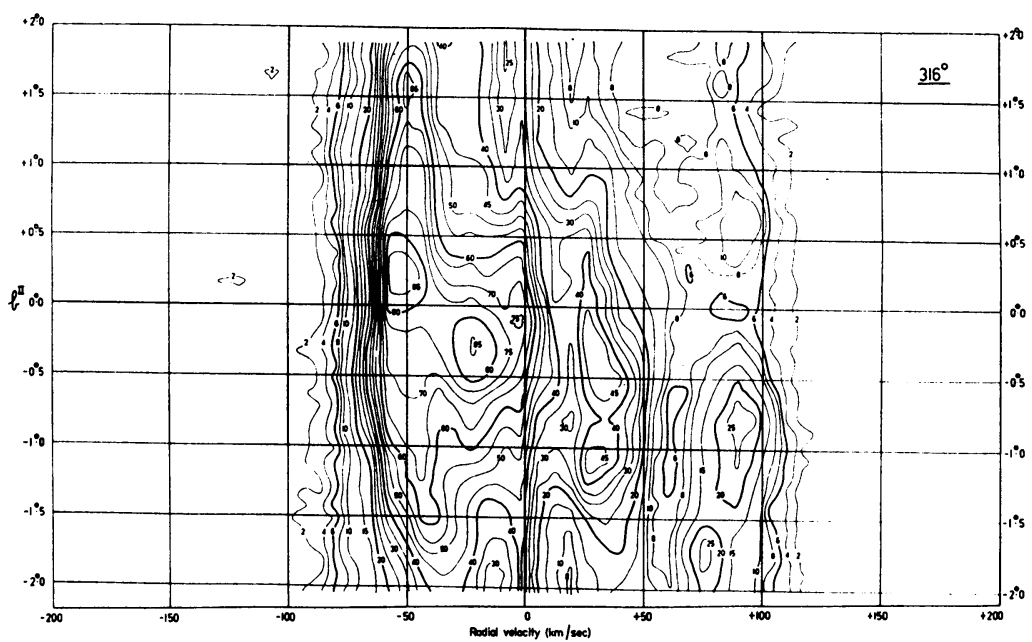

Fig. 4. Contour diagram in velocity-latitude plane for $l^{\mathrm{II}}=316^{\circ}(\mathrm{Kerr}, 1969 \mathrm{a})$.

ground, as was first pointed out by Mills (1958), and in the clustering of discrete sources, mainly Hil regions. The total flux in low-latitude sources is plotted as a function of longitude in Figure 5, from the observations of Beard et al. (1969).

In addition to the Norma-Scutum and Sagittarius arms, the Carina and Cygnus directions stand out as important concentrations of various constituents, both radio and optical. These results suggest that a Carina-Cygnus arm passes through the sun, but the continuity is difficult to establish in this nearby region. There appear to be fairly clear gaps in the pattern around longitudes $295^{\circ}$ and $60^{\circ}$.

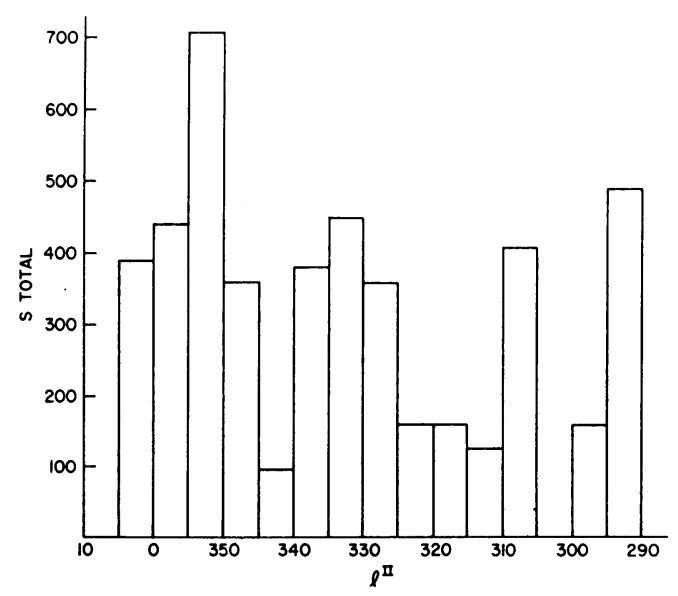

Fig. 5. Histogram of the total flux in discrete sources for $|b|<2^{\circ}$, as a function of longitude. (From observations of Beard et al., 1969.) 
It is well known that radio and optical spiral diagrams tend to look different, and it has often been suggested that gas and stars may be subject to different dynamical influences - in particular, that the gas may be significantly affected by magnetic or other nongravitational forces. We should therefore examine the relative kinematic properties of $\mathrm{HI}, \mathrm{HII}$ and stars.

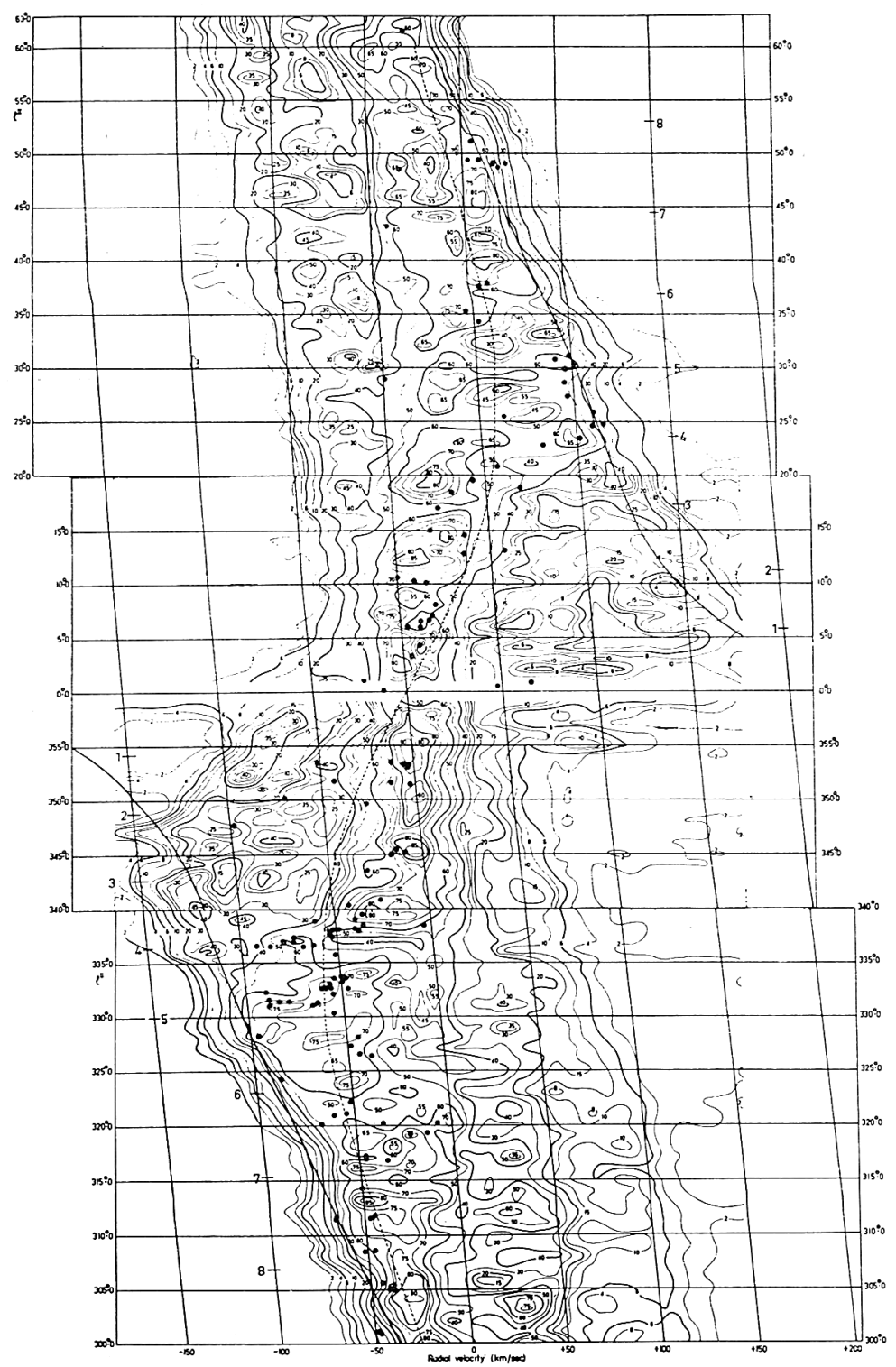

Fig. 6. Comparison between recombination-line velocities (black dots), measured by Mezger et al. (1970), and HI velocity-longitude contour plot (Kerr, 1969a). 


\section{HI and HII}

A relatively new radio approach to the spiral structure problem is through recombination line observations, which give evidence on the distribution and motions of $\mathrm{HII}$ regions. Dr. Mezger has given a detailed account of the radio work on Hil regions, but I will present some collaborative work on comparisons between the kinematical properties of $\mathrm{HI}$ and $\mathrm{HII}$.

Figure 6 shows such a velocity-longitude plot, to be published by Mezger et al. (1970), in which they superpose their HII observations from a joint NRAO-CSIRO survey at Parkes, Australia, on an Hi contour map from Parkes (Kerr, 1969a). There is a line of $\mathrm{HII}$ sources following the main ridge of local low-velocity $\mathrm{HI}$. The highervelocity Hir regions avoid the nuclear section, but otherwise they lie close to the $\mathrm{HI}$ ridges that are associated with the main spiral arms. Individual $\mathrm{H}$ II regions show their own peculiar velocities, but there is no sign of a systematic difference between $\mathrm{HI}$ and $\mathrm{H}$ II motions exceeding a few kilometers per sec.

An approximate rotation curve can also be derived for $\mathrm{H}$ II regions by fitting an upper envelope to the ensemble of the recombination line measures. This has been done by Mezger et al., who obtained a curve that is close to the HI rotation curve (Figure 7).

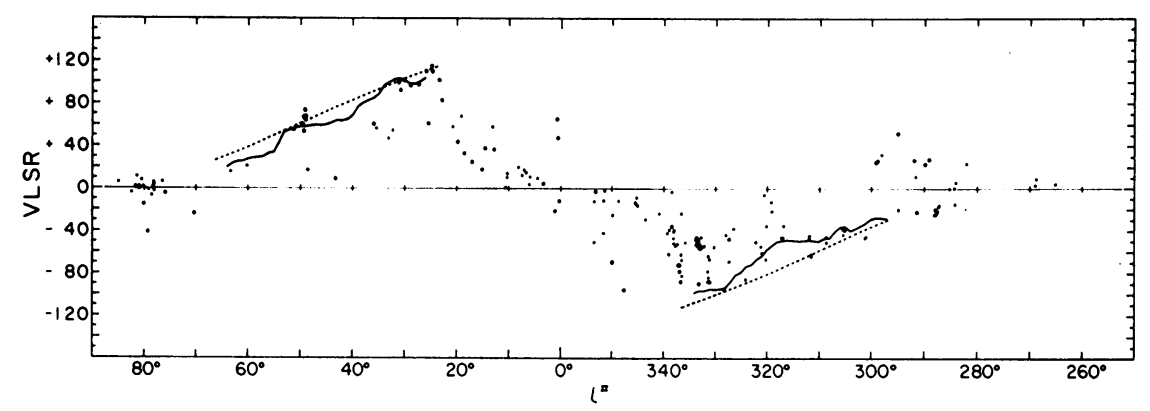

Fig. 7. Rotation curve derived from the upper envelope of the recombinationline velocities of Mezger et al. (1970).

Another type of $\mathrm{HI}_{\mathrm{I}} \mathrm{H}$ II comparison is obtained from a study of $21-\mathrm{cm}$ absorption, i.e., the absorption of continuum emission from $\mathrm{H}$ II regions in cooler $\mathrm{HI}$ clouds in the foreground. From such absorption observations, it is possible in many cases to gain information about the distances of $\mathrm{HII}$ regions which are too far away to be measurable optically. We cannot get precise absolute distances in this way, but we can place the $\mathrm{H}_{\mathrm{II}}$ regions in relation to the $\mathrm{HI}_{\mathrm{I}}$ emission features. Kerr and Knapp (1969) have shown that the HII regions can reasonably be located in this way in the main H I spiral arms.

Additional evidence comes from the velocities of absorption features in the 18-cm lines of $\mathrm{OH}$ and the $6-\mathrm{cm}$ line of $\mathrm{CH}_{2} \mathrm{O}$. These measures are not numerous enough to give an independent spiral picture, but the various types of velocity measures for 
gas in spiral arms are in approximate agreement. One direction in which we can expect good progress in the near future is in the detailed comparison of all the various types of measurement in as many directions as possible.

\section{Gas and Stars}

Several recent solar-motion solutions have been obtained with respect to the local neutral hydrogen, and all gave results that are close to the 'standard' solar motion, which is derived from the mean motion of the common stars. (For a detailed review, see Kerr, 1969b.) Solar-motion solutions with respect to the young Population I stars give results that differ a little from the standard solar motion, but such solutions depend greatly on selection effects and must be largely influenced by the irregularities of motion that occur over the several kiloparsecs in which the young stars can be seen.

Any systematic difference between the motions of gas and young stars cannot exceed $3-4 \mathrm{~km} \mathrm{~s}^{-1}$, which is the uncertainty produced by the irregularities in the regional motions in our vicinity. In regions of extreme irregularity, such as the portion of the Perseus arm studied by Rickard (1968) and Miller (1968), there are large departures from circular motion, but the significant result for the present discussion is that the $\mathrm{HI}, \mathrm{HII}, \mathrm{Ca}$ and stars all appear to be moving essentially together.

\section{Some Properties of $\mathrm{HI}_{\mathrm{I}}$ in Spiral Arms}

Although we cannot yet set up a definitive picture of the H I spiral pattern, there are a number of things we can say about the $\mathrm{HI}_{\mathrm{I}}$ in spiral arms.

Firstly, the $\mathrm{HI}_{\mathrm{I}}$ is clearly concentrated to spiral-like features. A major pattern can be seen, but there appears to be a high degree of splitting and interconnection of the main features. Even the major arms themselves show a longitudinal substructure in the form of variations of density and peculiar velocity, on a scale of about one kiloparsec. The regional velocity variations are in fact related to the density variations along an arm, as if each of the semi-discrete patches has its own peculiar velocity component. The Gould belt, the anomalous-velocity region of the Perseus arm, and the concentration of gas in the vicinity of W 51 may be considered as examples of three types of localized deviations from a smooth pattern.

One of the most important things that the observationalists can say to the theoreticians is that the pattern is highly irregular and fragmented, though certainly not as much so as in some of the pathological cases we can find in external systems. We should try to set up some measure of the 'degree of regularity' that we think we can see.

The density contrast between arms and interarm regions is an important quantity, but this is difficult to specify exactly in most of the Galaxy, because adjacent features tend to overlap each other on our contour maps. This arises from the internal velocity and latitude spread of the features themselves, and is not due to instrumental effects. In the few places where arms do stand out clearly, such as the regions outside the sun's distance from the center at longitudes around $40^{\circ}$ and $240^{\circ}$, there is a clear 
arm-interarm contrast of density of over 10 to 1 , but we do not know whether this degree of contrast exists in other parts of the Galaxy, especially in the inner part. One type of observation suggesting a low degree of contrast was first pointed out by Burke some years ago. In some parts of the Galaxy, the spiral arm peaks stand on top of a much broader background, as if there is a distributed component in addition to the arms. On the other hand, we often see matter apparently connected with the spiral arms, but extending a long way from the galactic plane. See for example the results of Goniadski in Argentina, quoted by Kerr (1969b).

The neutral hydrogen layer is very flat over most of the Galaxy, but measurable deviations are found for many of the spiral features. These are of the order of tens of parsecs, and the interesting thing is that a typical arm stays consistently above or below the plane for a considerable part of its length. This property assists in establishing the continuity of a particular feature (Henderson, 1967). Henderson, and also Varsavsky, have suggested that successive arms may be alternately above and below the plane, but I think the overall arrangement is probably not as regular as this.

Early $21-\mathrm{cm}$ results showed that the hydrogen layer is twisted in the outer parts in a systematic way, downwards on one side, and upwards on the other. We do not yet know for certain whether this effect is internally or externally produced, and thus whether it is relevant to the spiral structure problem. It is clear, however, that spiral structure is strongly established in these twisted outer regions. The normal relief map, which is based on the hydrogen centroid, suggests a gradual bending of a smooth layer, but this is an oversimplification. The hydrogen is strongly concentrated into arms, and in some places one sees two clearly distinct arms at about the same velocity, but at different latitudes. (Note that equality of velocity does not necessarily mean equality of distance.) An example is shown in Figure 8 (Hindman, 1969).

The hydrogen layer also broadens out considerably in the outer regions, as shown by Van Woerden and others. The thickness between half-density points increases from

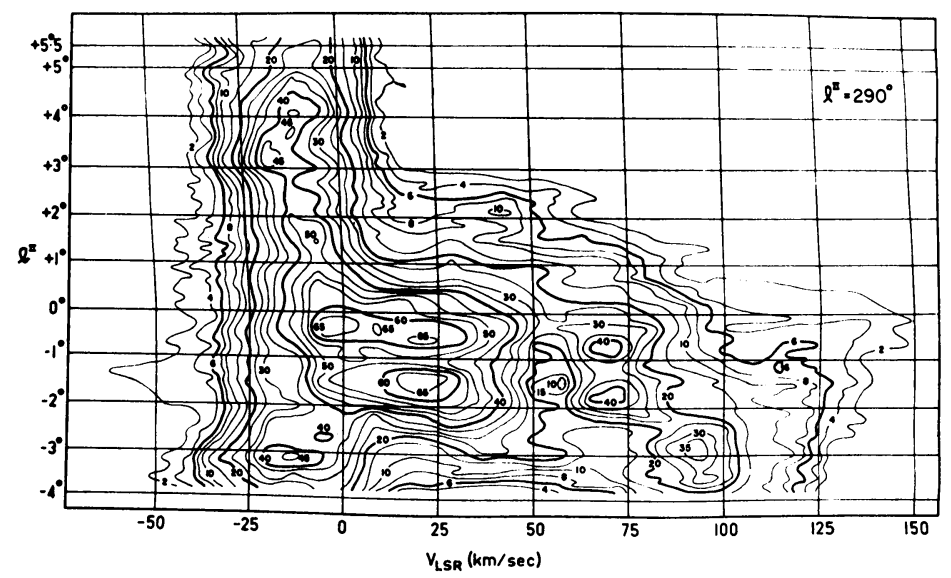

Fig. 8. Contour diagram in velocity-latitude plane for $l^{\mathrm{II}}=290^{\circ}$ (Hindman, 1969), showing the complexity of the spiral structure in the turned-down outer part of the Galaxy. 
$80-100 \mathrm{pc}$ near the center and $200 \mathrm{pc}$ in the solar region to 500 or $1000 \mathrm{pc}$ further out.

One of the interesting effects frequently found inside a spiral arm is an 'overturning motion'. For example, in the 3-kpc arm, the upper portion is approaching us more rapidly than the lower part, as first shown by Rougoor (1964). More recent data from the Parkes survey are given in Figure 9, which shows that the overturning motion

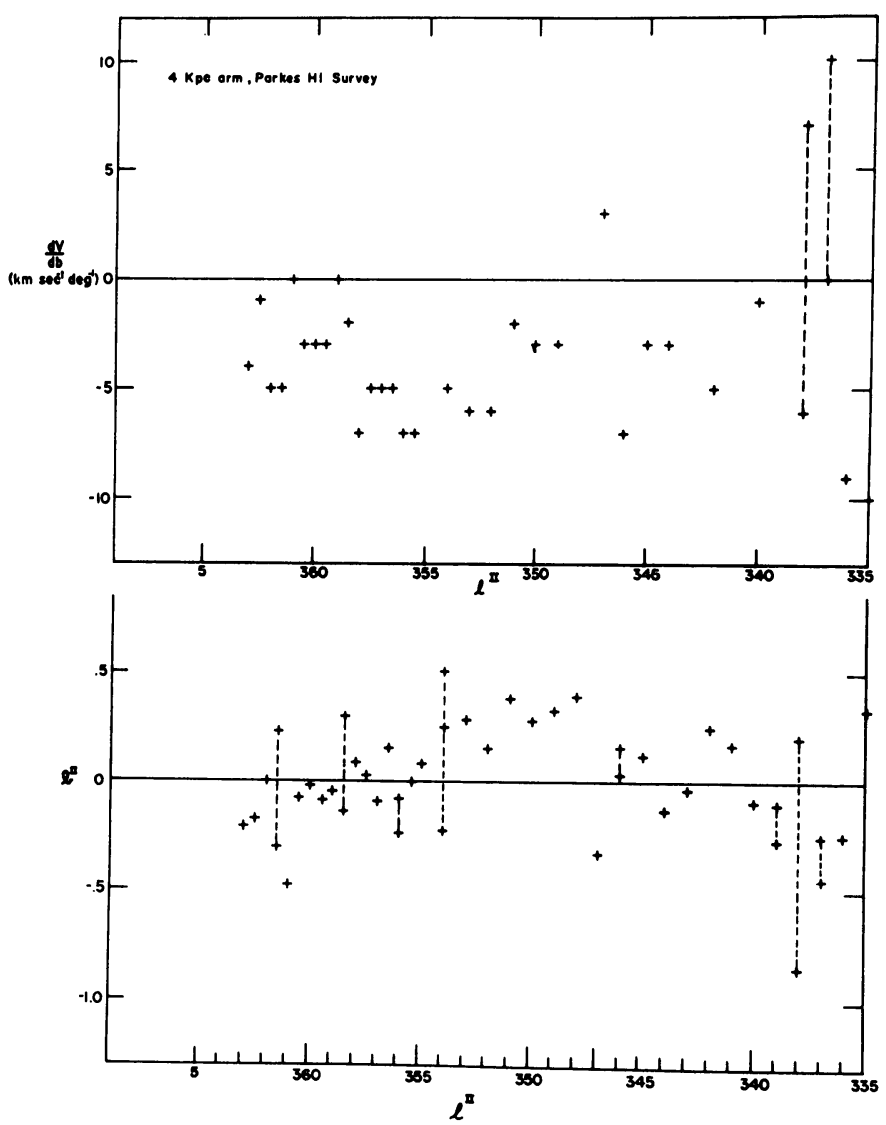

Fig. 9. The latitude and velocity gradient in the 3-kpc arm as a function of longitude, from Parkes survey data (Kerr, 1969a).

continues in the same sense over most of the longitude range in which we can see the arm. The diagram also shows the latitude of the arm, and there is a suggestion that the overturning is acting to bring the arm back into the galactic plane. Unfortunately, this is not always the case. An overturning motion is seen in many places, for example in the section of the Sagittarius arm shown in Figure 10, but there does not seem to be a systematic pattern. In several regions, the upper part is moving out from the center more quickly, but the reverse is also found. These effects may well be related to helical magnetic field patterns in spiral arms. 


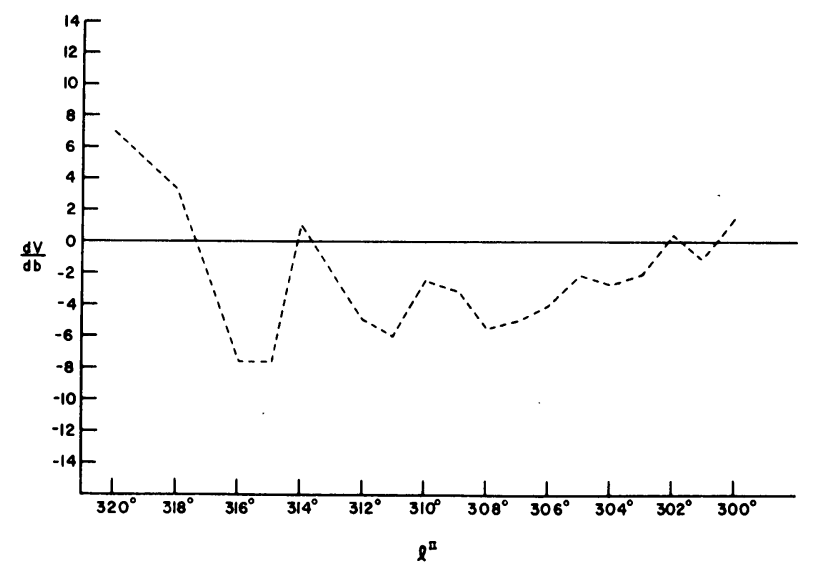

Fig. 10. Velocity gradient in part of the Sagittarius arm, from Parkes survey data.

\section{Symmetry}

I have placed great stress on irregularity. Another boundary condition to be placed on galactic models can come from considerations of symmetry. The velocity asymmetry has been discussed already. We have also studied the integrated hydrogen in various regions of the Galaxy and find some fairly clear results (Kaplan, unpublished).

(1) Inside the circle through the sun, the amount of hydrogen in the first and fourth quadrants is closely the same, except for a slight excess near the center in the fourth quadrant ('southern' side).

(2) The thickness of the hydrogen layer is noticeably larger in the fourth quadrant than the first in the general vicinity of the tangential point regions.

(3) Outside the solar circle, there is a distinct excess of hydrogen in the fourth quadrant over that in the first, and the effect persists at all latitudes in the disk layer.

These conclusions can be influenced by the velocity field and by optical depth considerations, but we have found no reasonable model to explain the observed results other than a real excess of material on one side over the other.

\section{Conclusion}

The 21-cm observations show clear evidence of a spiral pattern in the Galaxy, probably somewhere in the $\mathrm{Sb}-\mathrm{Sc}$ range. The detailed pattern is not yet clear, but a great deal of information is available on the characteristics and location of the spiral features.

\section{Acknowledgement}

This work has been supported by the U.S. National Science Foundation. 


\section{References}

Bajaja, E., Garzoli, S. L., Strauss, F., and Varsavsky, C. M.: 1967, IAU Symposium No. 31, p. 181. Beard, M., Day, G. A., and Thomas, B. M.: 1969, Australian. J. Phys., in press.

Henderson, A. P.: 1967, Ph.D. Thesis, University of Maryland.

Hindman, J. V.: 1969, in preparation.

Kerr, F. J.: 1964, IAU Symposium No. 20, p. 81.

Kerr, F. J.: 1969a, Australian. J. Phys. Astrophys. Suppl., No. 9, 1.

Kerr, F. J.: 1969b, Ann. Rev. Astron. Astrophys. 7, 39.

Kerr, F. J. and Knapp, G.: 1969, Australian. J. Phys. Astrophys. Suppl., in press.

Mezger, P. G., Wilson, T. L., Gardner, F. F., and Milne, D. K.: 1970, Astron. Astrophys. 4, 96.

Miller, J. S.: 1968, Astrophys. J. 151, 473.

Mills, B. Y.: 1958, IAU Symposium No. 9, p. 431.

Rickard, J. J.: 1968, Astrophys. J. 152, 1019.

Rougoor, G. W.: 1964, Bull. Astron. Inst. Netherl. 17, 381.

Shane, W. W. and Bieger Smith, G. P.: 1966, Bull. Astron. Inst. Netherl. 18, 263. 